\title{
Control of a Novel 5D Hyperchaotic System
}

\author{
Qiang Wei \\ Army Military Transportation University, China \\ Hong Niu \\ College of Electronic Information and Automation, \\ Tianjin University of Science \& Technology, China \\ E-mail:spots@163.com \\ www.tust.edu.cn
}

\begin{abstract}
In this paper, a novel five-dimensional (5D) autonomous hyperchaotic system is presented, and the characteristics of the 5D system are given in brief. For control of the 5D hyperchaotic system, a linear feedback controller is designed via the Lyapunov stability theory, so that the 5D system is no longer hyperchaotic but globally asymptotically converges to the equilibrium point at the origin. The numerical simulation results are given to illustrate the feasibility and effectiveness of the method.
\end{abstract}

Keywords: the novel 5D hyperchaotic system; hyperchaos control; Lyapunov stability theory; global asymptotic stability

\section{Introduction}

Hyperchaos was first proposed by Otto Rössler in 1979. ${ }^{1}$ Since then, many novel hyperchaotic systems have been formulated. In order to obtain hyperchaos, the system need to satisfy the following two important requisites. Firstly, the minimal dimension of the phase space that embeds a hyperchaotic attractor should be at least four, which requires the minimum number of coupled first-order autonomous ordinary differential equations to be four. Secondly, the number of terms in the coupled equations giving rise to instability should be at least two, of which at least one should have a nonlinear function. ${ }^{2}$ Therefore, hyperchaos is much more complicated than chaos, and it has greater engineering significance and application prospect in signal processing, secure communication and so on.

In this paper, a novel 5D hyperchaotic system, which has been introduced in Ref. 3, is reviewed. Stability control of the 5D system would be discussed, and some simulation results would be given to demonstrate the validity of the designed linear feedback controller.

\section{The Novel 5D Hyperchaotic System}

The dynamic equations of the novel 5D hyperchaotic system are formulated as

$$
\begin{aligned}
& \dot{x}=a(y-x), \\
& \dot{y}=(c-a) x+c y+w-x z, \\
& \dot{z}=-b z+x y, \\
& \dot{v}=m w, \\
& \dot{w}=-y-h v,
\end{aligned}
$$

where $x, y, z, v, w \in R$ are state variables, and $a=23$, $b=3, c=18, m=12$ and $h=4 .^{3}$

Let the initial values of the 5D system (1) be $\left(x_{0}, y_{0}\right.$, $\left.z_{0}, v_{0}, w_{0}\right)=(1,1,1,1,1)$, then the Lyapunov exponents 
respectively are $\lambda_{1}=0.8732>0, \lambda_{2}=0.1282>0, \lambda_{3}=$ $-0.0013 \approx 0, \lambda_{4}=-0.5770<0$ and $\lambda_{5}=-8.4231<0$. It indicates that the $5 \mathrm{D}$ system (1) is hyperchaotic. The
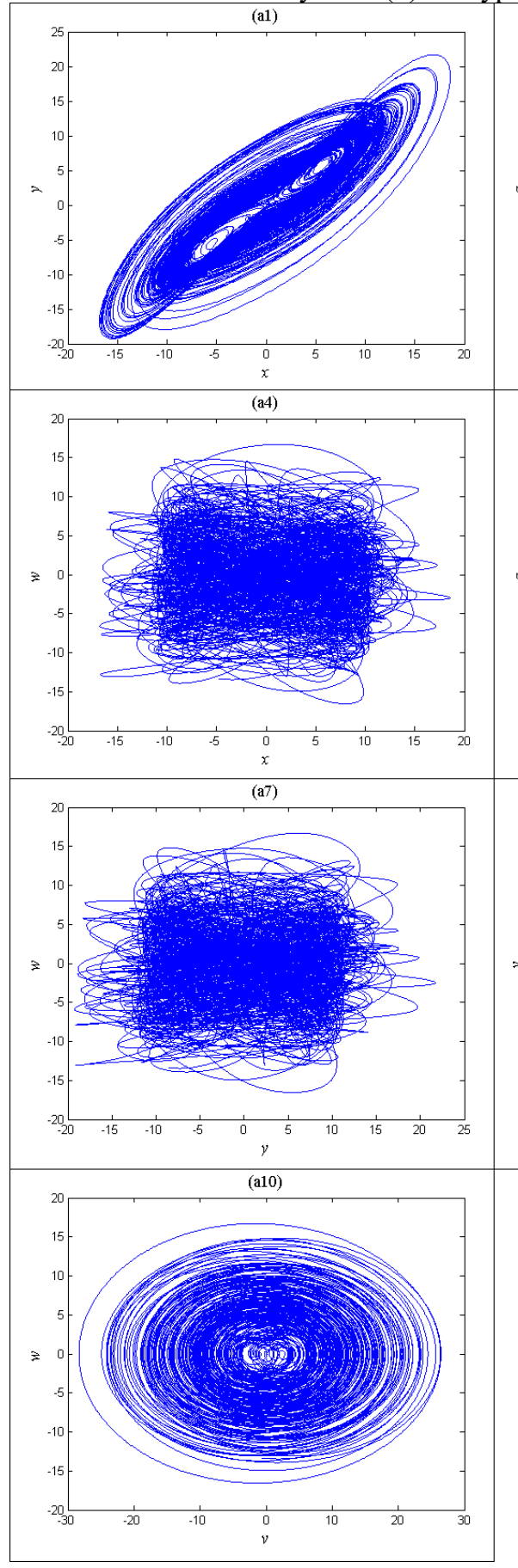

phase portraits of the 5D hyperchaotic system (1) are shown in Fig. 1. erchaotic. The

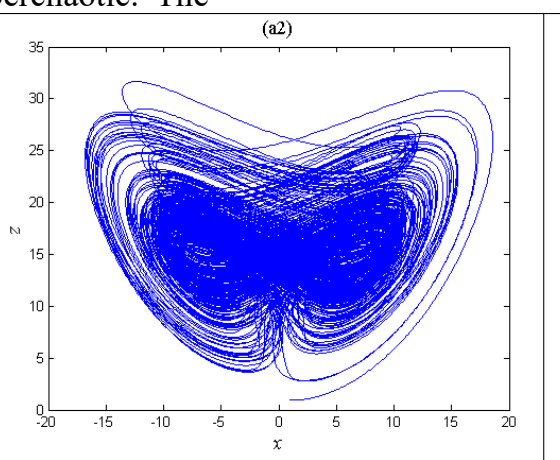

(a5)

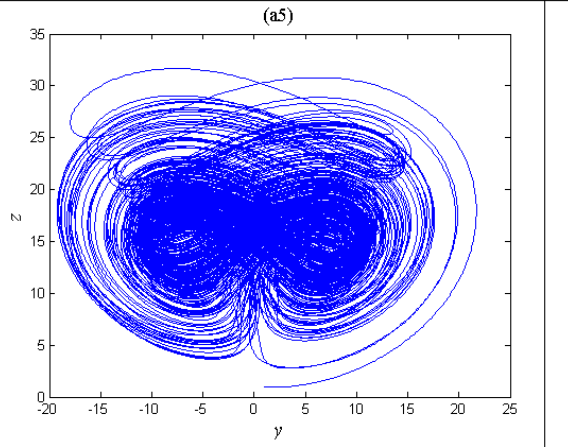

(a8)
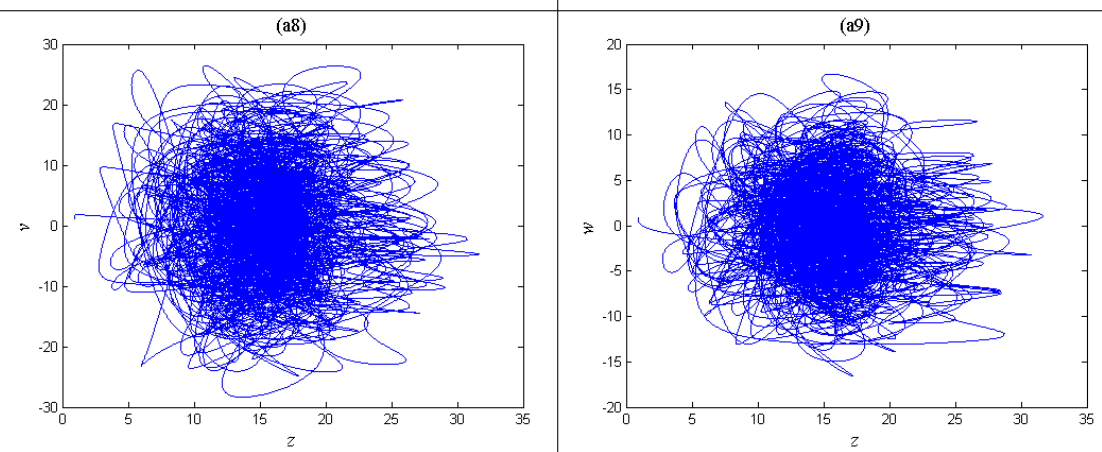

Fig. 1. Phase portraits of the 5D hyperchaotic system: (a1) $x-y$; (a2) $x-z$; (a3) $x-v$; (a4) $x-w$; (a5) $y-z$; (a6) $y-v$; (a7) $y-w$; (a8) $z-v$; (a9) $z-w ;($ a10) $v-w$ 


\section{Hyperchaos Control of the 5D System}

\subsection{Formulation of the controlled system}

The controlled system is represented as

$$
\begin{aligned}
& \dot{x}=a(y-x)+u_{c 1}, \\
& \dot{y}=(c-a) x+c y+w-x z+u_{c 2}, \\
& \dot{z}=-b z+x y+u_{c 3}, \\
& \dot{v}=m w+u_{c 4}, \\
& \dot{w}=-y-h v+u_{c 5},
\end{aligned}
$$

where

$$
\begin{aligned}
\boldsymbol{u}_{c} & =\left[\begin{array}{lllll}
u_{c 1} & u_{c 2} & u_{c 3} & u_{c 4} & u_{c 5}
\end{array}\right]^{\mathrm{T}} \\
& =\left[\begin{array}{lllll}
-k_{1} x & -k_{2} y & -k_{3} z & -k_{4} v & -k_{5} w
\end{array}\right]^{\mathrm{T}},
\end{aligned}
$$

and $k_{1}, k_{2}, k_{3}, k_{4}, k_{5} \geq 0$.

\subsection{Design of the linear feedback controller}

Theorem 1. Let $\boldsymbol{x}=\mathbf{0}$ be an equilibrium point for $\dot{\boldsymbol{x}}=\boldsymbol{f}(\boldsymbol{x})$, where $\boldsymbol{f}: D \rightarrow R^{n}$ is a locally Lipschitz map from a domain $D \subset R^{n}$ into $R^{n}$. Let $V: R^{n} \rightarrow R$ be a continuously differentiable function such that

$$
\begin{gathered}
V(\mathbf{0})=0 \text { and } V(\boldsymbol{x})>0, \quad \forall \boldsymbol{x} \neq \mathbf{0} \\
\|\boldsymbol{x}\| \rightarrow \infty \Rightarrow V(\boldsymbol{x}) \rightarrow \infty \\
\dot{V}(\boldsymbol{x})<0, \quad \forall \boldsymbol{x} \neq \mathbf{0}
\end{gathered}
$$

then $\boldsymbol{x}=\mathbf{0}$ is globally asymptotically stable. ${ }^{4}$

From Theorem 1, take a continuously differentiable function

$$
V=\frac{1}{2}\left(x^{2}+y^{2}+z^{2}+\frac{h}{m} v^{2}+w^{2}\right)
$$

as a Lyapunov function candidate for the controlled system (2). Then, the derivative $\dot{V}$ is given by

$$
\begin{aligned}
\dot{V}= & x \dot{x}+y \dot{y}+z \dot{z}+\frac{h}{m} v \dot{v}+w \dot{w} \\
= & -\left(k_{1}+a\right) x^{2}+c x y-\left(k_{2}-c\right) y^{2} \\
& -\left(k_{3}+b\right) z^{2}-k_{4} \frac{h}{m} v^{2}-k_{5} w^{2} \\
\leq & -\left(k_{1}+a-\frac{c}{2}\right) x^{2}-\left(k_{2}-\frac{3}{2} c\right) y^{2} \\
& -\left(k_{3}+b\right) z^{2}-k_{4} \frac{h}{m} v^{2}-k_{5} w^{2} .
\end{aligned}
$$

For $\dot{V}<0$, the parameters $k_{1}, k_{2}, k_{3}, k_{4}$ and $k_{5}$ should satisfy that

$$
\begin{aligned}
& k_{1}+a-\frac{c}{2}>0, \quad k_{1}>\frac{c}{2}-a, \quad k_{1}=0,
\end{aligned}
$$

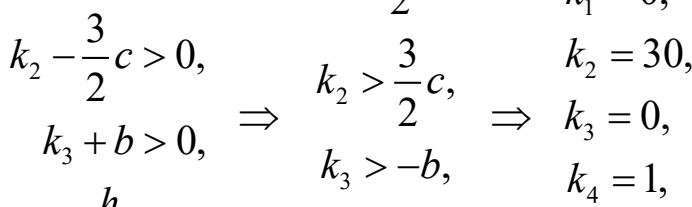

$$
\begin{aligned}
& k_{4} \frac{h}{m}>0, \quad k_{4}>0, \quad k_{5}=1 \text {. } \\
& k_{5}>0, \quad k_{5}>0 \text {, }
\end{aligned}
$$

As a result, the linear feedback controller $\boldsymbol{u}_{c}$ can be designed as

$$
\begin{aligned}
\boldsymbol{u}_{c} & =\left[\begin{array}{lllll}
u_{c 1} & u_{c 2} & u_{c 3} & u_{c 4} & u_{c 5}
\end{array}\right]^{\mathrm{T}} \\
& =\left[\begin{array}{lllll}
0 & -30 y & 0 & -v & -w
\end{array}\right]^{\mathrm{T}} .
\end{aligned}
$$

From Theorem 1, the controlled system (2) is globally asymptotically stable at the origin.

\subsection{Numerical simulation}

Let the initial values still be $\left(x_{0}, y_{0}, z_{0}, v_{0}, w_{0}\right)=(1,1,1$, $1,1)$, then the curves of the state variables of the controlled system (2) before and after adding the controller $\boldsymbol{u}_{c}$ are shown in Fig. 2 and Fig. 3 respectively. Comparing Fig. 3 with Fig. 2, it can be found that the state variables $x, y, z, v$ and $w$ converge to zero asymptotically and rapidly. It implies that the controlled system (2) is no longer hyperchaotic but asymptotically stable at the origin. It illustrates that the linear feedback controller $\boldsymbol{u}_{c}$ is feasible and effective for hyperchaos control of the $5 \mathrm{D}$ system (2).

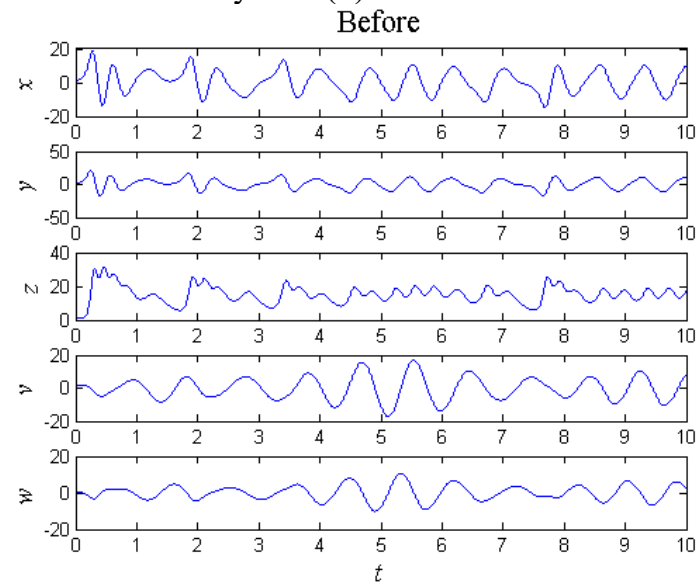

Fig. 2. Curves of the state variables of the controlled system before adding the controller $\boldsymbol{u}_{c}$

(C) The 2021 International Conference on Artificial Life and Robotics (ICAROB2021), January 21 to 24, 2021 


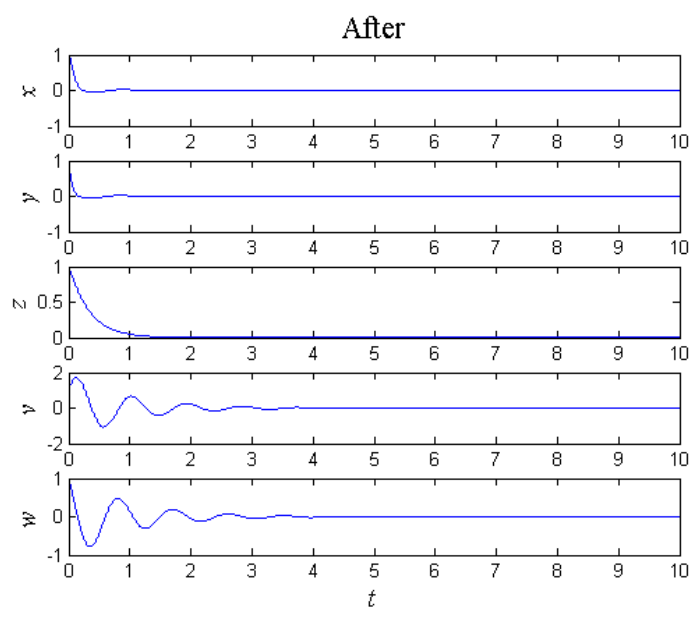

Fig. 3. Curves of the state variables of the controlled system after adding the controller $\boldsymbol{u}_{c}$

\section{Conclusions}

In this paper, a novel 5D hyperchaotic system is reviewed. For hyperchaos control of the 5D system, a linear feedback controller is designed. The numerical simulation results demonstrate the validity of the controller. The study in this paper has some engineering significance.

\section{References}

1. O. E. Rössler, An equation for hyperchaos, Physics Letters A 71(2-3), 1979, pp. 155-157.

2. T. G. Gao, G. R. Chen, Z. Q. Chen and S. J. Cang, The generation and circuit implementation of a new hyperchaos based upon Lorenz system, Physics Letters A 361(1-2), 2007, pp. 78-86.

3. Q. Wei and H. Niu, Analysis and circuit design of a novel 5D hyperchaotic system, Dynamical Systems and Control 8(2), 2019, pp. 118-128.

4. H. K. Khalil, Nonlinear Systems, 3rd edn., Prentice Hall, New Jersey, 2002, pp. 112-126. 